\title{
Multiuser communications with energy harvesting transmitters
}

\author{
Maria Gregori and Miquel Payaró \\ Centre Tecnològic de Telecomunicacions de Catalunya (CTTC) \\ 08860 - Castelldefels, Barcelona, Spain \\ E-mails: \{maria.gregori, miquel.payaro\}@cttc.cat
}

\begin{abstract}
This paper considers a communication system composed of multiple transmitter-receiver pairs (users) that must share the same frequency band. As battery replacement may be a tedious task, transmitters are solely powered by energy harvesters, specifically, by solar and electromagnetic energies. In previous works for non-harvesting nodes, transmissions of other nodes limit the achievable rate of a certain user due to interference. In contrast, when a certain transmitter harvests electromagnetic energy, transmissions of other nodes may be beneficial as part of the transmitted energy can be harvested and later used to increase the transmission power and, accordingly, the achievable rate. In this context, this paper formulates a Nash Equilibrium (NE) problem with prices to assign the transmission powers of the different nodes by taking into account constraints in the transmission power and the Energy Causality Constraints (ECCs), which intrinsically couple the different user transmission strategies. The existence and uniqueness of the equilibrium are characterized by using the theory of finite dimensional Variational Inequalities (VIs).
\end{abstract}

\section{INTRODUCTION}

Battery replacement is often a tedious task, specially, when nodes are placed in remote locations as usually occurs in wireless sensor networks. Energy Harvesting (EH) has become a potential technology to recharge the batteries of such nodes and thus to increase their autonomy. In this context, a node can be equipped with several EH modules from which different energy sources can be harvested, e.g., solar, wind or electromagnetic energy. The $\mathrm{EH}$ profile, i.e., the temporal variation of the harvested energy, depends on the type of harvester used by the node and affects its battery state, which ultimately limits its autonomy and performance. For example, the output of solar harvesters is usually constant during long periods of time, whereas, the power harvested by wind or electromagnetic harvesters may rapidly change due to the bursty nature of these energy sources [1].

When EH is considered, the traditional transmission strategies must be redesigned to deal with the temporal variations of the energy availability. This is usually enforced by ECCs, which impose that the cumulative energy spent by a node at a given time instant cannot be greater than the cumulative harvested energy at that same instant. Transmission strategies for $\mathrm{EH}$ nodes in point-to-point links were proposed in [2]-[5]. The problem of designing the power allocation that maximizes

This work was partially supported by: the Catalan Government under grant 2011FI_B 00956; the Spanish Ministry of Economy and Competitiveness under project TEC2011-29006-C03-01 (GRE3N-PHY); and the European Commission under project Network of Excellence in Wireless Communications (Newcom\#, Grant Agreement 318306). the transmission rate of an EH node on the interference channel was considered in [6] and [7] for the two-user Gaussian interference channel and the three-user cascade Gaussian $\mathrm{Z}$ interference channel. Wireless energy transfer was studied in [8] and references therein, where it was often assumed that the energy recipient is the receiver node.

Power control in multiuser communication systems was broadly studied for non-harvesting nodes, e.g., [9]-[12] and references therein. The authors of [9] showed that the sum-rate maximization in multiuser frequency selective Single-Input Single-Output (SISO) channels is an NP-hard problem. Consequently, different approaches were taken in the literature to deal with the nonconvexity of this problem: duality [10]; game theory [11]; and Pang et al. showed in [12] that the theory of finite-dimensional VIs [13] is the proper mathematical tool to deal with problems in which the user constraints are coupled, e.g., the temperature-interference constraints in cognitive radio systems.

Differently from the existing literature, this paper considers a multiuser communication system, which is critically affected by MultiUser Interference (MUI), where each transmitter is equipped with two EH modules. Specifically, we focus on the scenario where one of the sources is electromagnetic energy and the other source must satisfy that the incoming power is constant during sufficiently long periods of time, e.g., solar energy. As argued in Section II, the fact of considering the electromagnetic energy harvesters produces a coupling of the ECCs on the transmission powers of the different users since each node harvests part of the energy transmitted by the other nodes. Therefore, when a certain transmitter radiates power it has a positive and a negative impact on the achievable rates of the other users due to $\mathrm{EH}$ and interference, respectively.

\section{SYSTEM MODEL}

We consider a Gaussian interference channel composed of $\mathcal{T}$ transmitter and receiver pairs sharing the same band over SISO frequency-selective links composed of $K$ parallel subcarriers. We consider a time horizon of $N$ transmission blocks of duration $T_{s}$. The system model at a certain subcarrier $k, k=1, \ldots, K$, is exemplified in Fig. 1, where the channel power gain from transmitter $t$ to receiver $r$ at the $k$-th subcarrier is denoted by $h_{t r}(k)$ and, similarly, the gain from transmitter $t$ to transmitter $t^{\prime}$ at the $k$-th subcarrier is denoted by $\bar{h}_{t t^{\prime}}(k)$, which we consider reciprocal, i.e., $\bar{h}_{t t^{\prime}}(k)=$ $\bar{h}_{t^{\prime} t}(k)$. Throughout the paper, the term $t$-th user, $t \in[1, \mathcal{T}]$, denotes the link associated with the $t$-th transmitter-receiver 


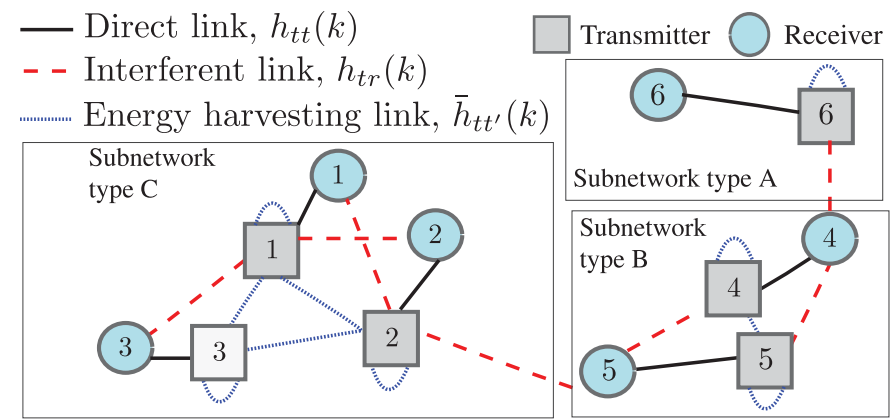

Fig. 1. Example of system model for $\mathcal{T}=6$. The harvesting sets are $\mathcal{H}_{1}=\mathcal{H}_{2}=\mathcal{H}_{3}=\{1,2,3\}, \mathcal{H}_{4}=\mathcal{H}_{5}=\{4,5\}$, and $\mathcal{H}_{6}=\{6\}$. Whereas, the interfering sets are $\mathcal{I}_{1}=\{2\}, \mathcal{I}_{2}=\mathcal{I}_{3}=\{1\}, \mathcal{I}_{4}=\{5,6\}$, $\mathcal{I}_{5}=\{2,4\}$, and $\mathcal{I}_{6}=\emptyset$. The subnetwork types are explained in Section V.

pair, which has an associated channel gain $h_{t t}(k)$. The set $\mathcal{I}_{t}$, $t=1, \ldots, \mathcal{T}$, contains the set of users that interfere user $t$.

As shown in Fig. 2, each transmitter is equipped with a battery, a supercapacitor and two EH modules that can harvest energy from two different sources (e.g., electromagnetic and solar energies). The power at the output of these harvesters is used to charge the supercapacitor. The energy in the supercapacitor is transferred to the battery at the end of each transmission block and, once the energy is in the battery, it can be used for transmission [14]. Thus, at the beginning of each transmission block, the battery of the $t$-th transmitter is charged with two energy packets that represent the contribution from each energy harvester. As it is mentioned above, the solar harvester provides a constant output power of $\frac{\beta_{t}}{T_{s}}$ Watts during the whole transmission duration. Thus, an energy packet of $\beta_{t}$ Joules is harvested at the beginning of each transmission block. The output power at the electromagnetic harvester can be obtained by multiplying the input power and the efficiency of the harvester that depends on the frequency of the incoming electromagnetic wave [15]. In this context, $\alpha_{t}(k)$ denotes the efficiency of the electromagnetic harvester in the $t$-th transmitter at the $k$-th subcarrier. The input power at the electromagnetic harvester depends on the transmission power of the nearby transmitters, $t^{\prime} \in \mathcal{H}_{t}$, where $\mathcal{H}_{t}$ contains the set of transmitters that contribute to charge the transmitter $t$, or, equivalently, the set of nodes that can charge from transmitter $t .{ }^{1}$ Without loss of generality, self-harvesting can be included in the model by letting $t$ belong to $\mathcal{H}_{t}$, which implies that part of the radiated energy of a certain transmitter can be harvested by its own harvesting module. ${ }^{2}$ Thus, the output power of the electromagnetic harvester of the $t$-th transmitter at the $n$-th time slot is $\sum_{t^{\prime} \in \mathcal{H}_{t}} \sum_{k=1}^{K} \alpha_{t}(k) \bar{h}_{t^{\prime} t}(k) P_{t^{\prime}}(k, n)$, where $P_{t^{\prime}}(k, n)$ denotes the transmission power of the transmitter $t^{\prime}$ at the $n$-th time slot and $k$-th subcarrier. For the mathematical analysis, we do not account energy that may be harvested from external transmissions (i.e., outside the studied network) and consider that the battery capacity is much larger than the

\footnotetext{
${ }^{1}$ Due to channel reciprocity, transmitter $t$ can harvest energy from transmitter $t^{\prime}$, if and only if the transmitter $t^{\prime}$ can harvest energy from the transmitter $t$.

${ }^{2}$ We have incorporated the self-harvesting as a possibility in the model; it is out of the scope of this paper to determine its practical feasibility.
}

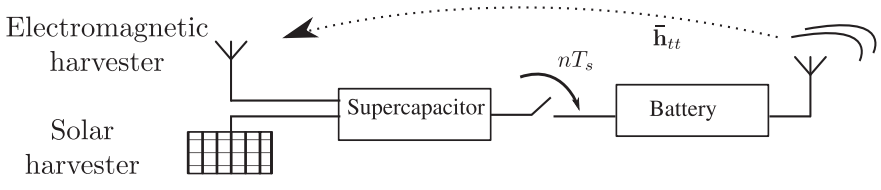

Fig. 2. Block diagram of the transmitter.

harvested energy so that no battery overflows are produced.

We consider that, for every user, a Gaussian distributed symbol is transmitted at each channel use and subcarrier. We do not consider interference cancellation techniques in order to avoid the need of having a centralized control or coordination in the network. Accordingly, we treat the MUI as additive colored noise. Thus, the rate of user $t$ depends on the radiated power of transmitter $t, \mathbf{p}_{t} \in \Re_{+}^{K N}, \mathbf{p}_{t}=\left(\left(P_{t}(k, n)\right)_{k=1}^{K}\right)_{n=1}^{N}$ and on the transmission power of all the transmitters that interfere $t$, i.e., $\mathbf{p}_{\mathcal{I}_{t}}$, and reads as

$r_{t}\left(\mathbf{p}_{t}, \mathbf{p}_{\mathcal{I}_{t}}\right)=\sum_{n=1}^{N} \sum_{k=1}^{K} \log \left(1+\frac{P_{t}(k, n) h_{t t}(k)}{\sigma_{t}^{2}(k)+\sum_{t^{\prime} \in \mathcal{I}_{t}} P_{t^{\prime}}(k, n) h_{t^{\prime} t}(k)}\right)$, where $\sigma_{t}^{2}(k)$ denotes noise power spectral density at the $t$-th receiver and $k$-th subcarrier.

A transmission strategy, which is composed of the radiated powers of the different users, i.e., $\mathbf{p}=\left(\mathbf{p}_{t}\right)_{t=1}^{\mathcal{T}}$, must satisfy certain conditions in order to be feasible. The local constraints, which can be ensured by each user independently of the actions undertaken by other users, are the temporalspectral mask constraints that limit the maximum transmitted power at a certain time instant and over a certain subcarrier (which may be enforced by radio regulatory bodies or by the maximum output power at the power amplifier), i.e., $\mathbf{p}_{t} \in \mathcal{P}_{t}$, where $\mathcal{P}_{t}=\left\{\mathbf{p}_{t} \in \Re^{K N}: \mathbf{0}_{K N} \preceq \mathbf{p}_{t} \preceq \mathbf{p}_{t}^{\max }\right\}$, with $\mathbf{p}_{t}^{\max }=\left(\left(p_{t}^{\max }(k, n)\right)_{k=1}^{K}\right)_{n=1}^{N}$. Let $\hat{\hat{\mathcal{P}}}=\prod_{t=1}^{\mathcal{T}} \mathcal{P}_{t}$ be the Cartesian product of the local domains of the $\mathcal{T}$ users, then the local constraints are ensured if $\mathbf{p} \in \hat{\mathcal{P}}$.

Additionally, the transmission strategy $\mathbf{p}$ must satisfy the ECCs (or coupled constraints), which impose that the battery level must be nonnegative. The remaining energy in the battery of transmitter $t$ by the end of the $n$-th slot is the difference between the harvested energy at the beginning of the slot and the expended energy by the end of the slot, i.e.,

$$
\begin{aligned}
& \phi_{t n}\left(\mathbf{p}_{t}, \mathbf{p}_{\mathcal{H}_{t}}\right) \triangleq \\
& \underbrace{\beta_{t} n+T_{s} \sum_{\ell=1}^{n-1} \sum_{k=1}^{K} \alpha_{t}(k) \sum_{t^{\prime} \in \mathcal{H}_{t}} P_{t^{\prime}}(k, \ell) \bar{h}_{t^{\prime} t}(k)}_{\text {Harvested energy }}-\underbrace{T_{s} \sum_{\ell=1}^{n} \sum_{k=1}^{K} P_{t}(k, \ell)}_{\text {Expended energy }},
\end{aligned}
$$

where $\mathbf{p}_{\mathcal{H}_{t}}$ contains the transmission powers of the transmitters that contribute to charge $t$ excluding $t$, i.e., $\mathbf{p}_{\mathcal{H}_{t}}=$ $\left\{\mathbf{p}_{t^{\prime}}: t^{\prime} \in \mathcal{H}_{t}, t^{\prime} \neq t\right\}$. Accordingly, $\mathbf{p}_{t}$ satisfies the ECCs if $-\phi_{t}\left(\mathbf{p}_{t}, \mathbf{p}_{\mathcal{H}_{t}}\right) \preceq \mathbf{0}_{N}$ with $\phi_{t}\left(\mathbf{p}_{t}, \mathbf{p}_{\mathcal{H}_{t}}\right)=\left(\phi_{t n}\left(\mathbf{p}_{t}, \mathbf{p}_{\mathcal{H}_{t}}\right)\right)_{n=1}^{N}$. Note that now the available set of strategies of a certain user depends on the strategies taken by the other users.

A certain power allocation $\mathbf{p}$ is feasible if the local and coupled constraints are satisfied for all users, i.e., $\mathbf{p} \in \mathcal{P}$ with 


$$
\mathcal{P}=\left\{\mathbf{p} \in \Re^{\mathcal{T} K N}: \mathbf{0}_{\mathcal{T} K N} \preceq \mathbf{p} \preceq \mathbf{p}^{\max },-\phi(\mathbf{p}) \preceq \mathbf{0}_{\mathcal{T} N}\right\},
$$

$\mathbf{p}^{\max }=\left(\mathbf{p}_{t}^{\max }\right)_{t=1}^{\mathcal{T}}$ and $\phi(\mathbf{p})=\left(\phi_{t}\left(\mathbf{p}_{t}, \mathbf{p}_{\mathcal{H}_{t}}\right)\right)_{t=1}^{\mathcal{T}}$, which is linear in the transmission power, i.e., $\phi(\mathbf{p})=\mathbf{A p}+\mathbf{b}$, where $\mathbf{A} \in \Re^{\mathcal{T} N \times \mathcal{T} K N}$ and $\mathbf{b} \in \Re^{\mathcal{T} N \times 1}$ can be obtained from (1).

There are different possible design goals, e.g., to determine the power allocation $\mathbf{p}$ that maximizes the overall network sum-rate, $\sum_{t=1}^{\mathcal{T}} r_{t}\left(\mathbf{p}_{t}, \mathbf{p}_{\mathcal{I}_{t}}\right)$. We do not tackle the sum-rate maximization because: it would require a centralized control; and the resulting problem is $N P$-hard as shown for the nonharvesting scenario in [9]. In this context, instead of studying the sum-rate maximization problem, we focus on the design of noncooperative distributed strategies. As it is commonly done in the literature, e.g., [12], to avoid the coupling among the user actions due to ECCs, we formulate the problem as a NE game with prices, where the objective function of each user depends on the achieved rate $r_{t}\left(\mathbf{p}_{t}, \mathbf{p}_{\mathcal{I}_{t}}\right)$ and on the prices, which may reward or penalize the user for using power in a certain time slot and subcarrier depending on the battery levels of the different nodes. If the prices are correctly chosen, the ECCs are fulfilled as presented in the following lines.

Accordingly, we introduce a tuple of prices $\lambda=$ $\left(\left(\lambda_{t n}\right)_{n=1}^{N}\right)_{t=1}^{\mathcal{T}}$, where $\lambda_{t n}$ imposes a penalty when the $n$-th ECC at the $t$-th transmitter is violated. The total penalty in the network is $\boldsymbol{\lambda}^{\top} \phi(\mathbf{p})=\boldsymbol{\lambda}^{\top}(\mathbf{A p}+\mathbf{b})=\gamma(\boldsymbol{\lambda})^{\top} \mathbf{p}+\boldsymbol{\lambda}^{\top} \mathbf{b}$, where $\boldsymbol{\gamma}(\boldsymbol{\lambda}) \triangleq \mathbf{A}^{\top} \boldsymbol{\lambda}=\left(\left(\left(\gamma_{t}(k, n)\right)_{k=1}^{K}\right)_{n=1}^{N}\right)_{t=1}^{\mathcal{T}^{T}}$,

$$
\gamma_{t}(k, n) \triangleq \underbrace{T_{s} \bar{\lambda}_{t n}}_{\begin{array}{c}
\text { Penalty for } \\
\text { using energy }
\end{array}}-\underbrace{T_{s} \sum_{t^{\prime} \in \mathcal{H}_{t}} \bar{\lambda}_{t^{\prime}(n+1)} \alpha_{t^{\prime}}(k) \bar{h}_{t t^{\prime}}(k)}_{\text {Reward for charging other nodes }},
$$

and $\bar{\lambda}_{t n}=\sum_{\ell=n}^{N} \lambda_{t \ell}$. Intuitively, $\gamma_{t}(k, n)$ can be understood as the net price to be paid for radiating power at transmitter $t$ in the $k$-th subcarrier of the $n$-th time slot (if $\gamma_{t}(k, n)<0$, a reward is obtained for radiating power). Note that when the $t$-th node transmits at the $n$-th time slot, it has an impact on the ECCs of the slots $n$ to $N$ : (i.) For the $t$-th node these constraints become more strict and this explains why, in (3), $\bar{\lambda}_{t n}$ adds up the constraint violation prices from $n$ to $N$; (ii.) For the nodes $t^{\prime} \in \mathcal{H}_{t} \backslash\{t\}$ the ECCs from $n+1$ to $N$ become less strict as the harvested energy can be used in these time slots. Thus, when the $t$-th node transmits at the $n$-th time slot, it has a reward obtained for charging other nodes that depends on the channel gain between the two transmitters $\bar{h}_{t t^{\prime}}(k)$, the efficiency of the electromagnetic harvester at the $k$-th subcarrier, $\alpha_{t^{\prime}}(k)$, and the addition of the constraint violation prices from $n+1$ to $N, \bar{\lambda}_{t^{\prime}(n+1)}, \forall t^{\prime} \in \mathcal{H}_{t}$.

Therefore, we study the following NE game

$$
\begin{aligned}
\max _{\mathbf{p}_{t}} & r_{t}\left(\mathbf{p}_{t}, \mathbf{p}_{\mathcal{I}_{t}}\right)-\sum_{n=1}^{N} \sum_{k=1}^{K} P_{t}(k, n) \gamma_{t}(k, n) \\
\text { subject to } & \mathbf{p}_{t} \in \mathcal{P}_{t},
\end{aligned}
$$

for all $t=1, \ldots, \mathcal{T}$, with an additional complementary condition over the constraint violation prices $\lambda_{t n}$ that enforces the fulfillment of the ECCs, i.e., $\forall t=1, \ldots, \mathcal{T}, \forall n=1, \ldots, N$,

$$
0 \leq \lambda_{t n} \perp \phi_{t n}\left(\mathbf{p}_{t}, \mathbf{p}_{\mathcal{H}_{t}}\right) \geq 0,
$$

where $\mathbf{0} \preceq \mathbf{a} \perp \mathbf{b} \succeq \mathbf{0}$ stands for $\mathbf{a}^{\top} \mathbf{b}=0, \mathbf{a} \succeq \mathbf{0}$, and $\mathbf{b} \succeq \mathbf{0}$.

The temporal-spectral mask constraints are imposed in (4b), whereas, the ECCs are satisfied thanks to the complementary condition in (5). Summarizing, the remainder of the paper aims at finding a tuple $\left\{\mathbf{p}^{\star}, \boldsymbol{\lambda}^{\star}\right\}$ that jointly solves the NE problem in (4) and satisfies the complementary condition in (5). In the next section, we focus on solving the NE problem in (4) for a given price tuple, whereas in Section IV, we will address the problem of finding the price tuple that satisfies the complementary condition in (5).

Remark 1. The system model is general enough to cover the following scenarios: (i.) some transmitters are only equipped with a single harvester, e.g., set $\alpha_{t}(k)=0, \forall k$, if there is only a solar harvester or set $\beta_{t}=0$ when a certain node only has an electromagnetic harvester; (ii.) some nodes are connected to the power grid and do not have energy limitations due to the ECCs. For these nodes, set $\beta_{t}=T_{s} \max _{n} \sum_{k=1}^{K} p_{t}^{\max }(k, n)$.

\section{The NE PROBLEM WITH GIVEN PRICES}

In this section, we use the finite VI theory to show that the $\mathrm{NE}$ of the game in (4) always exists and that, under certain conditions, the NE is unique for any price tuple $\gamma$, which can be computed from the constraint violation prices $\lambda$ and (3).

In the following, we denote the NE problem in (4) for a given price tuple $\gamma$ as $\mathcal{G}_{\gamma}$. The NE of $\mathcal{G}_{\gamma}$ is a tuple of strategies $\mathbf{p}^{\gamma}=\left[\mathbf{p}_{1}^{\gamma \top}, \ldots, \mathbf{p}_{\mathcal{T}}^{\gamma^{\top}}\right]^{\top}$ such that, $\forall t \in[1, \mathcal{T}]$, we have

$$
r_{t}\left(\mathbf{p}_{t}^{\gamma}, \mathbf{p}_{\mathcal{I}_{t}}^{\gamma}\right)-\gamma_{t}^{\top} \mathbf{p}_{t}^{\gamma} \geq r_{t}\left(\mathbf{p}_{t}, \mathbf{p}_{\mathcal{I}_{t}}^{\gamma}\right)-\gamma_{t}^{\top} \mathbf{p}_{t}, \quad \forall \mathbf{p}_{t} \in \mathcal{P}_{t} .
$$

To determine the existence and uniqueness properties of the NE of $\mathcal{G}_{\gamma}$, we first define the vector function $\mathbf{f}(\mathbf{p}): \Re_{+}^{K N T} \rightarrow$ $\Re^{K N T}$ with $\mathbf{f}(\mathbf{p})=\left(\mathbf{f}_{t}(\mathbf{p})\right)_{t=1}^{\mathcal{T}}, \mathbf{f}_{t}(\mathbf{p}) \triangleq-\nabla_{\mathbf{p}_{t}} r_{t}\left(\mathbf{p}_{t}, \mathbf{p}_{\mathcal{I}_{t}}\right)=$ $\left(\left(f_{t}(k, n)\right)_{k=1}^{K}\right)_{n=1}^{N}$ and

$$
f_{t}(k, n)=-\left(\frac{\sigma_{t}^{2}(k)+\sum_{t^{\prime} \in \overline{\mathcal{I}}_{t}} P_{t^{\prime}}(k, n) h_{t^{\prime} t}(k)}{h_{t t}(k)}\right)^{-1},
$$

where $\overline{\mathcal{I}}_{t}=\mathcal{I}_{t} \cup\{t\}$.

Remark 2. The authors of [12] were the first to study the interference channel under a VI approach and derived sufficient conditions for $\mathbf{f}(\mathbf{p})$ to be a uniformly $P$-function and a strongly monotone function (the definitions are given in [13, Definitions 3.5.8 and 2.3.1]). As proved by Pang et al. in [12], $\mathbf{f}(\mathbf{p})$ is a uniformly $P$-function if all the users receive or generate low MUI and $\mathbf{f}(\mathbf{p})$ is a strongly monotone function if all the users receive and generate low MUI. These two properties of $\mathbf{f}(\mathbf{p})$ are of a great importance to characterize the existence and uniqueness of the NE as shown next.

Lemma 1. The $N E$ game $\mathcal{G}_{\gamma}$ in (4): (i.) is equivalent to $\operatorname{VI}(\hat{\mathcal{P}}, \mathbf{f}(\mathbf{p})+\gamma)$; (ii.) always admits one solution; and (iii.) has a unique solution if $\mathbf{f}(\mathbf{p})$ is a uniformly $P$-function on $\hat{\mathcal{P}}$.

Proof: The proof of (i.) follows directly from the theory of VIs [13, Section 1.4.2]. To prove the existence and uniqueness 


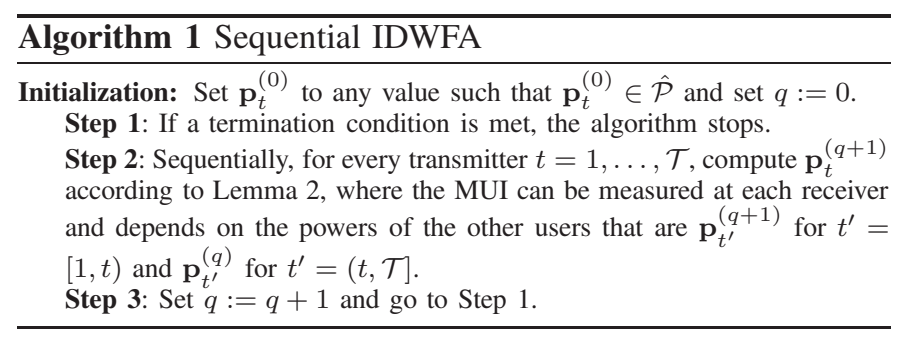

properties of the NE, we use the VI reformulation of the NE problem. The existence is guaranteed from [13, Corollary 2.2.5] by noting that the set $\hat{\mathcal{P}}$ is compact and convex and the function $\mathbf{f}(\mathbf{p})$ is continuous. Finally, (iii.) is guaranteed by [13, Proposition 3.5.10], where one must note that if $\mathbf{f}(\mathbf{p})$ is a continuous uniformly $P$-function, so it is $\mathbf{f}(\mathbf{p})+\gamma$.

Additionally, the following lemma characterizes the structure of the transmission powers at the NE, i.e., $\mathbf{p}_{t}^{\gamma}=$ $\left(\left(P_{t}(k, n)^{\gamma}\right)_{k=1}^{K}\right)_{n=1}^{N}$ :

Lemma 2. In every $N E$, the transmission power of the $t$-th user is given by the following Directional Water-Filling (DWF) expression:

$P_{t}(k, n)^{\boldsymbol{\gamma}}=\left[\frac{1}{\gamma_{t}(k, n)}-\frac{\sigma_{t}^{2}(k)+\sum_{t^{\prime} \in \mathcal{I}_{t}} P_{t^{\prime}}(k, n) h_{t^{\prime} t}(k)}{h_{t t}(k)}\right]_{0}^{P_{t}^{\max }(k, n)}$

if $\gamma_{t}(k, n) \geq 0$ and $P_{t}(k, n)^{\gamma}=P_{t}^{\max }(k, n)$ if $\gamma_{t}(k, n)<0$.

Proof: In the NE, each of the users solves the maximization problem in (4). As (4) is a strictly convex program, the solution is unique and can be derived from the KKT optimality conditions as given in Lemma 2.

The main difference of the power allocation in (8) with respect to the well known Iterative Waterfilling Algorithm (IWFA) [12], is that in (8) the water level, i.e., $\frac{1}{\gamma_{t}(k, n)}$, varies over the time and frequency dimensions (the temporal variations are due to $\mathrm{EH}$ and the frequency variations are due to the frequency-selectivity of the channel gains and efficiencies of the electromagnetic harvesters). Thus, from Lemmas 1 and 2 , if $\mathbf{f}(\mathbf{p})$ is a continuous uniformly $P$-function, $\mathcal{G}_{\gamma}$ has a unique NE that can be found, e.g., by means of the sequential Iterative Directional Waterfilling Algorithm (IDWFA), which is presented in Algorithm 1. Note that Algorithm 1 proceeds as the IWFA [12], but accounting for the temporal and frequency variations of the water level. If $\mathbf{f}(\mathbf{p})$ is a continuous uniformly $P$-function, the sequential IDWFA guarantees convergence, which can be proved following the same approach as in [12, Theorem 6] and is omitted for brevity.

IV. Optimal PRices AT THE NASH Equilibrium of $\mathcal{G}$

In this section, we solve the problem of determining a tuple $\left\{\mathbf{p}^{\star}, \boldsymbol{\lambda}^{\star}\right\}$ that jointly solves the NE problem in (4) and satisfies the complementary condition in (5), which is denoted as problem $\mathcal{G}$.

Lemma 3. (i.) The problem $\mathcal{G}$ is equivalent to $\operatorname{VI}(\mathcal{P}, \mathbf{f}(\mathbf{p}))$, which always admits, at least, one solution. (ii.) If $\mathbf{f}(\mathbf{p})$ is a continuous uniformly $P$-function, $\mathcal{G}$ is equivalent to the following Nonlinear Complementary Problem (NCP):

$$
\operatorname{NCP}(\bar{\phi}): \mathbf{0}_{\mathcal{T} N} \preceq \boldsymbol{\lambda} \perp \overline{\boldsymbol{\phi}}(\boldsymbol{\lambda}) \succeq \mathbf{0}_{\mathcal{T} N},
$$

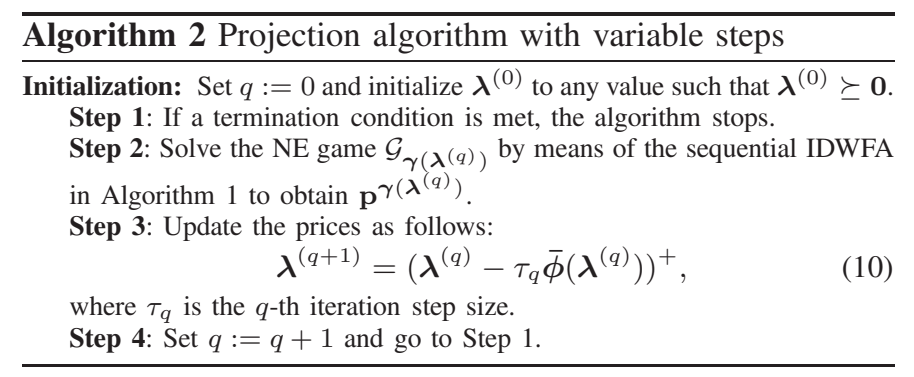

where $\bar{\phi}(\boldsymbol{\lambda}): \Re_{+}^{\mathcal{T} N} \rightarrow \Re^{\mathcal{T} N}$ is a map defined as $\bar{\phi}(\boldsymbol{\lambda})=$ $\mathbf{A p}^{\gamma(\boldsymbol{\lambda})}+\mathbf{b}$, with $\mathbf{p}^{\gamma(\boldsymbol{\lambda})}$ being the unique NE of the problem $\mathcal{G}_{\boldsymbol{\gamma}(\boldsymbol{\lambda})}$ in (4) given the prices $\gamma(\boldsymbol{\lambda})$. (iii.) If $\mathbf{f}(\mathbf{p})$ is a strongly monotone function, then the solution to $\operatorname{VI}(\mathcal{P}, \mathbf{f}(\mathbf{p}))$ is unique.

Proof: The NE problem in (4) can be expressed with the KKT conditions: $0 \leq P_{t}(k, n) \perp f_{t}(k, n)+\gamma_{t}(k, n)+\mu_{t k n} \geq$ $0 \forall t, k, n$ and $0 \leq \mu_{t k n} \perp\left(p_{t}^{\max }(k, n)-P_{t}(k, n)\right) \geq 0 \forall t, k, n$, where $\mu_{t k n}$ is the multiplier associated with the temporalspectral mask constraint of user $t$ in the $k$-th subcarrier and $n$-th time slot. These equations along with the complementary condition in (5) form a mixed nonlinear complementary problem that is equivalent to the KKT system of $\operatorname{VI}(\mathcal{P}, \mathbf{f}(\mathbf{p}))$, which follows from [13, Proposition 1.3.4]. From [13, Corollary 2.2.5], $\operatorname{VI}(\mathcal{P}, \mathbf{f}(\mathbf{p}))$ has at least one solution, which proves (i.). To prove (ii.), let $\left\{\mathbf{p}^{\mathcal{G}}, \lambda^{\mathcal{G}}\right\}$ be a NE of $\mathcal{G}$. Then, $\lambda^{\mathcal{G}}$ must satisfy the complementary condition in (5), i.e., $\mathbf{0} \leq \boldsymbol{\lambda}^{\mathcal{G}} \perp \boldsymbol{\phi}\left(\boldsymbol{\lambda}^{\mathcal{G}}\right) \geq \mathbf{0}$. Additionally, as $\mathbf{f}(\mathbf{p})$ is a uniformly Pfuncion, $\mathbf{p}^{\mathcal{G}}$ can be obtained as the unique equilibrium of the NE problem in (4) where the prices are $\gamma\left(\lambda^{\mathcal{G}}\right)$. Conversely, let $\lambda^{\mathrm{NCP}}$ be a solution to the $\operatorname{NCP}(\bar{\phi})$ in $(9)$, then the tuple $\left\{\mathbf{p}^{\mathrm{NCP}}, \lambda^{\mathrm{NCP}}\right\}$ is an equilibrium of $\mathcal{G}$, where $\mathbf{p}^{\mathrm{NCP}}$ is the unique equilibrium of (4) where the prices are $\gamma\left(\boldsymbol{\lambda}^{\mathrm{NCP}}\right)$. From [13, Theorem 2.3.3] and the strong monotonicity of $\mathbf{f}(\mathbf{p})$, it directly follows that the $\mathrm{NE}$ of $\mathcal{G}$ is unique, which proves (iii.).

In the following, we focus on scenarios in which $\mathbf{f}(\mathbf{p})$ is strongly monotone (i.e., from Remark 2, low-received and lowgenerated MUI) in order to determine the unique $\mathrm{NE}$ of the game $\mathcal{G}$. To do so, we solve the $\operatorname{NCP}(\bar{\phi})$ in (9) instead of directly solving the $\operatorname{VI}(\mathcal{P}, \mathbf{f}(\mathbf{p}))$ because the domain of the NCP is the nonnegative orthant and the solution can be found in a computationally efficient manner. There exist several algorithms in the literature to solve monotone problems, c.f., [13, Chapter 12]. Among them, we use a projection algorithm with variable steps that is summarized in Algorithm 2 and guarantees convergence if $0<\tau_{q} \leq 2 c_{\operatorname{coc}}(\bar{\phi}), \forall q$, where $c_{c o c}(\bar{\phi})=\frac{c_{s m}(\mathbf{f})}{\|\mathbf{A}\|_{2}^{2}}$ is the co-coercive constant of $\bar{\phi}(\boldsymbol{\lambda}), c_{s m}(\mathbf{f})$ is the strong monotonicity constant of $\mathbf{f}(\mathbf{p})$ [12], $\mathbf{A}$ is a $\mathcal{T} N \times \mathcal{T} K N$ matrix that linearly relates the transmission power with the ECCs and $\|\cdot\|_{2}$ denotes the induced matrix norm. The convergence proof can be derived similarly to the proof of [12, Theorem 10] and is omitted for brevity.

\section{DISCUSSION ON THE NETWORK IMPLEMENTATION}

The solution derived in the previous section guarantees that the network operates at the NE as far as the solar irradiation profile does not vary significantly along time. We want that, 
when a significant change in the solar irradiation is observed, the network is able to adapt autonomously so that each node operates at the transmission power that guarantees the NE given the new values of the solar harvesting powers. In this context, this section discuses about the different possible implementations, i.e., centralized or distributed, and the required signaling in each of the cases.

Centralized scheme: During the network deployment phase, it is possible to compute in a centralized manner the NE of $\mathcal{G}$ for the different possible solar EH profiles of all nodes (using Algorithm 2). Then, each node contains a table in local memory that stores its transmission powers at the NE for the different solar irradiation profiles of the remaining nodes. In this context, when a substantial change on the solar irradiation is observed, each of the nodes solely needs to broadcast its solar harvested energy, $\beta_{t}$, collect the solar harvested energies of other nodes, $\boldsymbol{\beta}$, and check in the tables which is the transmission power at the NE to adjust its transmission power.

Distributed scheme: At the cost of some extra signaling, it is also possible to have a distributed scheme to compute the NE. At any transmission block, each transmitter has the following information: (a.) the solar and electromagnetic harvested energies, i.e., $\beta_{t}$ and $T_{s} \sum_{k=1}^{K} \alpha_{t}(k) \sum_{t^{\prime} \in \mathcal{H}_{t}} P_{t^{\prime}}(k, \ell) \bar{h}_{t^{\prime} t}(k)$, $\forall n$, respectively, which can be measured at the output of the harvesters; (b.) the remaining energy in the battery $\phi_{t n}\left(\mathbf{p}_{t}, \mathbf{p}_{\mathcal{H}_{t}}\right), \forall n$, which can be locally measured; and (c.) the MUI, $\sum_{t^{\prime} \in \mathcal{I}_{t}} P_{t^{\prime}}(k, n) h_{t^{\prime} t}(k), \forall k, n$, which can be measured at the respective receiver and forwarded by a feedback link.

Note that, in order to solve Step 2 of Algorithm 2 at any given iteration $q$, the only nonlocal additional information required by user $t$ is $\gamma_{t}^{(q)}(k, n), \forall k, \forall n$, which is given in (3). This can be computed by the $t$-th transmitter if it knows: (i.) the efficiency of the electromagnetic harvesters of the nodes in $\mathcal{H}_{t}$, i.e., $\alpha_{t^{\prime}}(k), \forall k, \forall t^{\prime} \in \mathcal{H}_{t}$; (ii.) the channel gains $\bar{h}_{t t^{\prime}}(k)$, $\forall t^{\prime} \in \mathcal{H}_{t}$; (iii.) its own constraint violation prices $\lambda_{t n}^{(q)}, \forall n$; and (iv.) the constraint violation prices of the nodes in $\mathcal{H}_{t} \backslash\{t\}$, i.e., $\lambda_{t^{\prime} n}^{(q)}, \forall n, \forall t^{\prime} \in \mathcal{H}_{t} \backslash\{t\}$.

Any node can have the information in (i.) and (ii.) as it is static. The price updates in (iii.) can be computed from the previous prices $\lambda_{t n}^{(q-1)}, \forall n$, by using (10), where the energy in the battery in each of the time slots is known as argued in (b.) above. Then, the critical information required at each transmitter to have a complete distributed solution is the price updates of the other nodes, (iv.). In a general network topology, computing (iv.) may not be possible due to the limited local information in each node and, in such networks, it is necessary that each transmitter broadcasts its prices $\boldsymbol{\lambda}_{t}$ to the transmitters $t^{\prime} \in \mathcal{H}_{t}$ so that all the nodes in the network can have a complete knowledge of (iv.). However, there may be some specific network topologies in which this signaling is not required. To characterize these network topologies, we first define the following three subnetwork types (see Fig. 1):

Type A: Network in which the transmitters do not harvest energy from other nodes, i.e., $\mathcal{H}_{t} \subseteq\{t\}, \forall t$. Type B: Network composed of two transmitter-receiver pairs in which the two transmitters charge each other, i.e., $\mathcal{H}_{t}=\{1,2\}, \forall t=1,2$. Type C: Network composed of three transmitter-receiver pairs such that energy can only be harvested from nodes within the subnetwork and no interference is produced from other subnetworks, i.e., $\mathcal{H}_{t}, \mathcal{I}_{t} \subseteq\{1,2,3\}, \forall t=1, \ldots, 3$. Additionally, where for each node $t$, the $2 \times 2$ matrix $\left[h_{t_{1} t}(k) h_{t_{2} t}(k) ; \bar{h}_{t_{1} t}(k) \bar{h}_{t_{2} t}(k)\right]$ is full rank, $\forall k$, where $t_{1}$ and $t_{2}$ are the indices of the other nodes in the subnetwork.

Lemma 4. When the network is composed of the union of subnetworks type $A, B$, and $C$ and such that $\mathbf{f}(\mathbf{p})$ is strongly monotone, then the unique $N E$ of $\mathcal{G}$ can be computed in a distributed manner using Algorithm 2 without the need of any signaling between nodes throughout the algorithm iterations.

Proof: (iv.) can be recursively computed from (10) if each transmitter $t$ is able to estimate the remaining energy in the battery of transmitters $t^{\prime} \in \mathcal{H}_{t}$ or, equivalently, to estimate $P_{t^{\prime}}(k, n), \forall t^{\prime} \in \mathcal{H}_{t} \backslash\{t\}$. For subnetworks type A, the set $\mathcal{H}_{t} \backslash\{t\}$ is empty and thus (iv.) is not required. For subnetworks type $\mathrm{B}, P_{t^{\prime}}(k, n), t^{\prime} \neq t$, can be estimated by solving the equation obtained from the electromagnetic harvested energy. Whereas in subnetworks type $\mathrm{C}$, an additional equation is needed due to the additional unknown. This additional equation can be obtained from the MUI.

As mentioned in the previous section, the ECCs are satisfied once Algorithm 2 converges to $\lambda^{\star}$. In future work, we will study the evolution of the ECCs through the iterations of Algorithm 2 and analyze how its convergence is affected when some node completely depletes its battery.

\section{NUMERICAL RESUltS}

In this section, we numerically evaluate and compare the rates obtained in two different network topologies composed of $\mathcal{T}=3$ users as depicted in Fig. 3. We consider $K=10$ subcarriers with bandwidth $1 \mathrm{MHz}$ and central frequency $2401+k \mathrm{MHz}, k \in[1, K]$ (IEEE 802.15.1), and $N=20$ transmission blocks of duration $T_{s}=1 \mathrm{~s}$. As shown in Table I, we consider three different case studies, namely, (CS.1), (CS.2), and (CS.3), in which we vary the EH capabilities of the network nodes: in (CS.1), the three nodes harvest both electromagnetic and solar energies; in opposition, (CS.2) considers that all the network nodes are solely powered by solar energy; finally, (CS.3) considers that the transmitter $t=1$ is connected to the power grid and that the other nodes are powered both by the solar and electromagnetic harvesters. We have set $p_{t}^{\max }(k, n)=2.5 \mathrm{~mW}, \forall t, k, n$ and considered that the channel attenuation is due to path loss, where we have used the Friis equation with the distances depicted in Fig. 3. The near field radiation pattern has been considered for the self charging channel gain, i.e., $\bar{h}_{t t}(k), \forall t$ [16]. The transmitters and receivers have directive antennas with an antenna gain of $4 \mathrm{~dB}$ in the direct link; $2 \mathrm{~dB}$ in the links with the other $\mathrm{EH}$ transmitters; and $-1 \mathrm{~dB}$ gain in all the other directions.

For this setup, Fig. 4 shows the rate achieved at the NE by means of Algorithm 2 for the different topologies and case studies. In the simulations, it is observed that the users 

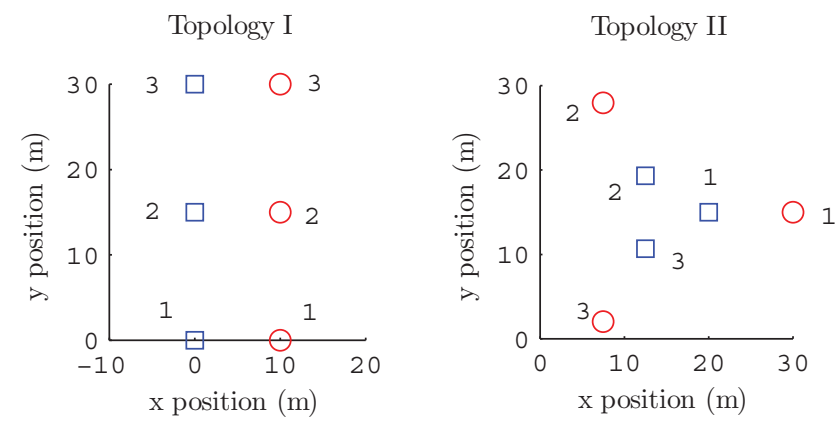

Fig. 3. Considered topologies. The squares and circles denote the position of the transmitter and receivers, respectively.

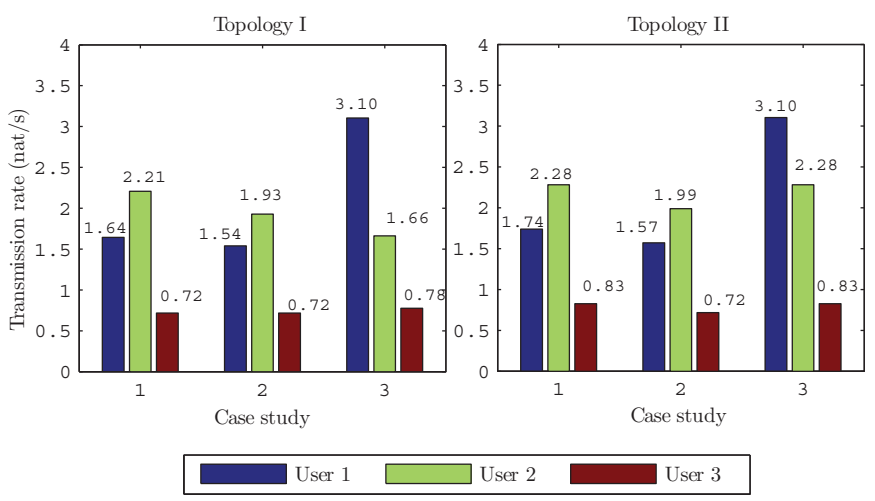

Fig. 4. Transmission rate at the NE for the different users, case studies and topologies.

with a higher solar harvested power also achieve a higher rate as the solar power is the major source of energy in the node. However, the electromagnetic harvester also provides a relevant source of energy as it is noted by comparing case studies (CS.1) and (CS.2), in which a significant rate decrease is produced when the transmitters are not equipped with the electromagnetic harvester. This decrease is $6.24,12.64$, and $0 \%$ for users 1 to 3 in Topology I and 9.70, 12.82, and $13.20 \%$ in Topology II, respectively. In (CS.3), we observe that when user 1 is connected to the power grid, it experiences a tremendous increase in its rate since its transmission power is only limited by the local constraints (the ECC are trivially satisfied). This increase in the transmission power of user 1 may be beneficial or disadvantageous for the other nodes depending on the system parameters, e.g., the channel gains $\bar{h}_{1 t^{\prime}}(k)$ and $h_{1 r}(k), t^{\prime}, r \in[2,3]$. Additionally, we have observed that each user tends to allocate power in the subcarriers in which the efficiency of its own energy harvester, $\alpha_{t}(k)$, is greater. This is because the self charging channel gain $\bar{h}_{t t}(k)$ is greater than the gains for charging other users $\bar{h}_{t t^{\prime}}(k), t^{\prime} \neq t$, due to the associated link distances.

\section{CONCLUSION}

Energy harvesting establishes a new paradigm in power allocation strategies for multiuser communication systems since transmissions of other nodes not always penalize the achievable rate, but they may indeed be beneficial thanks to the additional energy availability. In this work, we have studied the noncooperative NE problem by taking into account both
Table I

PARAMETERS USED IN THE DIFFERENT CASE STUDIES.

\begin{tabular}{l|c|c|c}
\hline Case study & $t=1$ & $t=2$ & $t=3$ \\
\hline (CS.1-2) & $\beta_{1}=12 \mathrm{~mJ}$ & $\beta_{2}=16 \mathrm{~mJ}$ & $\beta_{3}=6 \mathrm{~mJ}$ \\
(CS.3) & Power grid & $\beta_{2}=16 \mathrm{~mJ}$ & $\beta_{3}=6 \mathrm{~mJ}$ \\
\hline (CS.1,3) & $\alpha_{1}(k)=0.1 k$ & $\alpha_{2}(k)=1.1-0.1 k$ & $\alpha_{3}(k)=0.5$ \\
(CS.2) & $\alpha_{1}(k)=0, \forall k$ & $\alpha_{2}(k)=0, \forall k$ & $\alpha_{3}(k)=0, \forall k$ \\
\hline
\end{tabular}

temporal-spectral mask constraints and the ECCs. We have seen that, under certain conditions, there exists a unique NE that can be distributedly computed by the network nodes. We have numerically shown that the solar harvester is the main energy source of the nodes; however, a significant rate increase is produced thanks to the electromagnetic harvester. By using node cooperation, the achievable rates may further increase at a cost of an additional signaling, which will be further studied in subsequent works.

\section{REFERENCES}

[1] C. K. Ho, P. D. Khoa, and P. C. Ming, "Markovian models for harvested energy in wireless communications," in IEEE International Conference on Communication Systems, 2010, pp. 311-315.

[2] C. K. Ho and R. Zhang, "Optimal energy allocation for wireless communications with energy harvesting constraints," IEEE Trans. Signal Process., vol. 60, no. 9, pp. 4808-4818, Sep. 2012.

[3] O. Ozel, K. Tutuncuoglu, J. Yang, S. Ulukus, and A. Yener, "Transmission with energy harvesting nodes in fading wireless channels: Optimal policies," IEEE J. Sel. Areas Commun., vol. 29, no. 8, pp. 1732-1743, 2011.

[4] M. Gregori and M. Payaró, "Energy-efficient transmission for wireless energy harvesting nodes," IEEE Trans. Wireless Commun., vol. 12, no. 3, pp. 1244-1254, 2013.

[5] —, "On the precoder design of a wireless energy harvesting node in linear vector gaussian channels with arbitrary input distribution," IEEE Trans. Commun., vol. 61, no. 5, pp. 1868-1879, 2013.

[6] X. Liu and E. Erkip, "Transmission schemes for gaussian interference channels with transmitter processing energy," IEEE Trans. Wireless Commun., vol. 12, no. 9, pp. 4756-4765, 2013.

[7] K. Tutuncuoglu and A. Yener, "Sum-rate optimal power policies for energy harvesting transmitters in an interference channel," Journal of Communications and Networks, vol. 14, no. 2, pp. 151-161, 2012.

[8] L. Liu, R. Zhang, and K.-C. Chua, "Wireless information transfer with opportunistic energy harvesting," IEEE Trans. Wireless Commun., vol. 12, no. 1, pp. 288-300, 2013.

[9] Z.-Q. Luo and S. Zhang, "Dynamic spectrum management: Complexity and duality," IEEE J. Sel. Topics Signal Process., vol. 2, no. 1, pp. 5773, 2008.

[10] W. Yu and R. Lui, "Dual methods for nonconvex spectrum optimization of multicarrier systems," IEEE Trans. Commun., vol. 54, no. 7, pp. 1310$1322,2006$.

[11] G. Scutari, D. P. Palomar, and S. Barbarossa, "Asynchronous iterative water-filling for gaussian frequency-selective interference channels," IEEE Trans. Inf. Theory, vol. 54, no. 7, pp. 2868-2878, 2008.

[12] J.-S. Pang, G. Scutari, D. P. Palomar, and F. Facchinei, "Design of cognitive radio systems under temperature-interference constraints: A variational inequality approach," IEEE Trans. Signal Process., vol. 58, no. 6, pp. 3251-3271, 2010.

[13] F. Facchinei and J.-S. Pang, Finite-dimensional variational inequalities and complementarity problems. Springer, 2003.

[14] L. Berbakov, C. Antón-Haro, and J. Matamoros, "Optimal transmission policy for cooperative transmission with energy harvesting and battery operated sensor nodes," Signal Processing, vol. 93, no. 11, pp. 3159$3170,2013$.

[15] J. Hagerty, F. Helmbrecht, W. McCalpin, R. Zane, and Z. Popovic, "Recycling ambient microwave energy with broad-band rectenna arrays," IEEE Trans. Microw. Theory Tech., vol. 52, no. 3, pp. 1014-1024, 2004.

[16] A. F. Molisch, K. Balakrishnan, C.-C. Chong, S. Emami, A. Fort, J. Karedal, J. Kunisch, H. Schantz, U. Schuster, and K. Siwiak, "Ieee 802.15. 4a channel model-final report," IEEE P802, vol. 15, no. 04, p. 0662, 2004. 